\title{
Monitoring of Blood Pressure is not Enough to Avoid Neonatal Postoperative Encephalopathy
}

\author{
Simón Pedro Lubián López, MD, PhD ${ }^{1}$ Amaya Zuazo Ojeda, $\mathrm{MD}^{2}$ Gema Jimenez Gómez, PhD 3 \\ Isabel Benavente Fernández, MD, PhD ${ }^{1}$ \\ ${ }^{1}$ Neonatology Department, "Puerta del Mar” University Hospital, Cadiz, \\ Spain. Fundación Nene (Neonatal Neurology Research Group \\ 2 Radiology Department, "Puerta del Mar" University Hospital, Cadiz, Spain \\ ${ }^{3}$ Research Unit, "Puerta del Mar" University Hospital, Cadiz, Spain \\ Address for correspondence Isabel Benavente Fernández, MD, PhD, \\ Neonatology Department, "Puerta del Mar" University Hospital, \\ $3^{\text {a }}$ planta, Avenida Ana de Viya 21, 11009 Cadiz, Spain \\ (e-mail: isabenavente@gmail.com).
}

Am J Perinatol Rep 2018;8:e192-e194.

\begin{abstract}
Background Neonatal encephalopathy with seizures after general anesthesia not occurring in infants undergoing cardiac or major neurosurgery is very uncommon. An ischemic origin due to cerebral hypoperfusion from perioperative hypotension has been suggested, but there is a lack of a consensus definition for intraoperatory hypotension in neonates.

Case Report We report the first case of neonatal encephalopathy with seizures in a neonate with anorectal malformation. He underwent a colostomy with caudal

Keywords

- encephalopathy

- postoperative

- hypotension

- caudal anesthesia

- diffusion-weighted imaging

- neonate anesthesia combined with light general anesthesia. Intraoperative systolic blood pressure and mean blood pressure values were considered normal. Thirty-two hours after the intervention, the patient presented electroclinical seizures. Diffusionweighted imaging showed bilateral involvement with reduced diffusivity in the watershed areas of the middle cerebral artery and posterior cerebral artery.

Conclusion Perioperative monitoring of blood pressure is not enough in neonatal surgery. Cerebral magnetic resonance imaging should be considered in infants with noncardiac congenital anomalies after neonatal surgery and long-term follow-up is required.
\end{abstract}

Neonatal encephalopathy with seizures after general anesthesia unrelated to local anesthetic toxicity or not occurring in infants undergoing cardiac or major neurosurgery, is very uncommon and its cause is unknown in most of cases. ${ }^{1}$ An ischemic origin due to cerebral hypoperfusion from perioperative hypotension has been suggested. ${ }^{1,2}$ Mean blood pressure (MBP) is considered a good marker of cerebral perfusion but the physiological blood pressure range that allows adequate cerebral perfusion remains unknown in neonates.

In response to the concern of potential neurotoxicity in children, the use of caudal anesthesia combined with light general anesthesia is common and is considered not to be associated with hypotension in neonates.

Diffusion-weighted imaging (DWI) is a useful clinical tool to assess the extent and pattern of injury in hypoxiaischemia-related brain injury. Moderate and prolongated

received

January 22, 2017

accepted after revision

February 15, 2017
DOI https://doi.org/

$10.1055 / \mathrm{s}-0038-1668565$. ISSN 2157-6998. hypotension with impaired autoregulation is considered to be one of the underlying mechanisms in the pathogenesis of predominant watershed injury in magnetic resonance. ${ }^{3}$

Brain injury after anorectal malformation neonatal surgery is very uncommon ${ }^{2}$ and, to our knowledge, it has not been described under caudal anesthesia.

We present the case of a neonate who presented with seizures due hypoxia-ischemia-related brain injury demonstrated in DWI following caudal anesthesia to perform a colostomy.

\section{Case Study}

A male child born at 39 gestational weeks (birth weight, $3.5 \mathrm{~kg}$ ), after noncomplicated vaginal delivery. Previous cardiotocographic monitoring was reassuring, and there was no need for

Copyright @ 2018 by Thieme Medical Publishers, Inc., 333 Seventh Avenue, New York, NY 10001, USA Tel: +1(212) 584-4662.
License terms

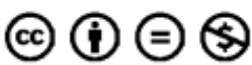


resuscitation after delivery as the Appearance, Pulse, Grimace, Activity, and Respiration scores were 9 and 10 in minutes 1 and 5, respectively. Umbilical cord pH 7.31. Anorectal malformation was noted after birth. The infant was hemodynamically and neurologically stable before surgery with baseline systolic blood pressure (SBP) and MBP readings of 60 and $40 \mathrm{~mm} \mathrm{Hg}$, respectively. Before undergoing surgery, his blood glucose levels were $92 \mathrm{mg} / \mathrm{dL}$ and he was breathing spontaneously with $\mathrm{pCO}_{2} 41 \mathrm{~mm} \mathrm{Hg}$ and oxygen saturation of $97 \%$.

At 36 hours of life, he underwent a colostomy with caudal anesthesia ( $1 \mathrm{~mL} / \mathrm{kg}$ levobupivacaine $0.125 \%$ ) combined with light general anesthesia (sevoflurane, $6 \%$ inspired concentration with a $100 \%$ fraction of inspired oxygen, and one dose of $10 \mathrm{mg}$ propofol) with no episodes of adverse events. Intraoperative records using an automated oscillometric method revealed SBP values of between 40 and $60 \mathrm{~mm} \mathrm{Hg}$ and MBP values of 28 and $35 \mathrm{~mm} \mathrm{Hg}$. There were no episodes of hypoxemia, hypocapnia, hypoglycemia, or hyperthermia, neither during surgery nor in the neonatal intensive care unit postoperatively.

Thirty-two hours after the intervention, the patient presented recurrent clonic movements of the upper and lower right limb with lethargy. Clinical seizures were recorded by amplitude-integrated electroencephalography (aEEG) (Brainz Instrument Ltd., New Zealand) and the background pattern was continuous normal voltage. Seizures were controlled with one dose of phenobarbital, and it did not recur and the blood glucose level was normal at that time. Four days after seizures, the child underwent cerebral magnetic resonance imaging (MRI) without sedation which in DWI showed bilateral involvement with reduced diffusivity in the watershed areas of the middle cerebral artery and posterior cerebral artery with involvement of the splenium of the corpus callosum and sparing of the central gray matter (-Fig. 1). High signal intensity in T2- and T1-weighted images through parietal-occipital cortical gray matter and subcortical white matter was also reported.

At 1 year of age, neurological status assessed by the Hammersmith Infant Neurological Examination and neurodevelopmental assessment using the Bayley Scales of Infant and Toddler Development, Third Edition, were normal.

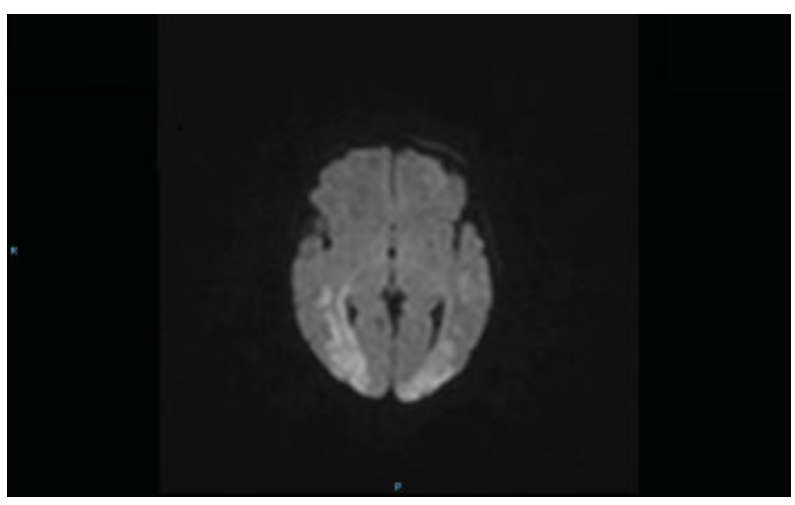

Fig. 1 Diffusion-weighted imaging. Bilateral involvement with reduced diffusivity in the watershed areas of the middle cerebral artery and posterior cerebral artery.

\section{Discussion}

Infants who have neonatal surgery for noncardiac congenital anomalies are at risk of neurodevelopmental delay. ${ }^{2}$ The etiology of neurodevelopmental impairments is currently unknown, but several potential risk factors have been proposed. Among the mechanisms of central nervous system injury in infants having noncardiac surgery may include hypoxic ischemia, although this has not been well characterized.

Impaired cerebral autoregulation has been observed in full-term infants receiving anesthesia and the hypoxic ischemia injury may be attributable to cerebral hypoperfusion. Inadequate perfusion from hypotension can lead to partial asphyxia and we know that partial ischemia often causes damage in the watershed areas between major cerebral blood vessels. $^{3}$

It is widely believed that caudal anesthesia has limited hemodynamic effects. However, studies measuring cerebral blood flow have reported a decrease in blood flow after caudal block. In the context of combined general and regional anesthesia, hypotension can be observed, although this is not widely reflected in the literature.

On the other hand, there is a lack of a consensus definition for intraoperatory hypotension in neonates with great variability in the threshold used for defining and treating intraoperatory hypotension. A MBP of $17 \mathrm{~mm} \mathrm{Hg}$ is considered as -2 standard deviation for neonates undergoing anesthetized procedures. ${ }^{4}$ The lowest SBP of patients with severe postoperative encephalopathy in the case series by McCann et $\mathrm{al}^{1}$ ranged from 22 to $40 \mathrm{~mm} \mathrm{Hg}$. In our case, intraoperative readings of SBP and MBP ranged between 40 and 60 and 28 and $35 \mathrm{~mm} \mathrm{Hg}$, respectively. Therefore, we believe that with current normally considered blood pressure thresholds may underestimate the risk of postoperative encephalopathy.

DWI enables the detection of brain lesions in babies within the first hours after a hypoxic ischemic insult because they can depict a water mobility impairment before any signal intensity changes on T1- or T2-weighted images occur. DWI is, therefore, a sensitive magnetic resonance technique for determining the pattern of brain injury and the time of hypoxic ischemic insult with normalization of this sequence at 7 days after insult. The watershed pattern of injury seen is typically thought to follow "prolonged partial asphyxia" and moderate hypotension. ${ }^{3}$ Posterior watershed involvement, with sparing of the anterior areas, has been observed in cases of neonatal encephalopathy following hypoxia ischaemia ${ }^{5}$ and symptomatic hypoglycemia. Hypoxic-ischemic injury in a posterior watershed distribution are not invariably associated with an adverse outcome, ${ }^{5}$ but these infants may develop cerebral visual impairment, cognitive delay, epilepsy, learning difficulties, as well as behavioral, concentration, and memory problems, and they must be followed until school age.

In other cases of postoperative encephalopathy, it was difficult to establish the time of insult and etiology. We know that in states of hypoxia ischemia the seizures appear between 18 and 48 hours after the insult and DWI findings appear 
between the first hours and 7 days after a hypoxic-ischemic insult. In our case, hypoglycemia was excluded and the chronology of seizures, starting 32 hours after the intervention, and findings at cerebral magnetic resonance performed 4 days later suggest that the time of insult could be related with surgery.

In other case series, most of the infants had preoperative neurologic issues, were preterm infants, or required major neonatal surgery. ${ }^{1}$ Our case was a term newborn, neurologically stable before surgery.

We consider perioperative monitoring of blood pressure is not enough in neonatal surgery and we believe that it is necessary to monitor cerebral oxygenation with near-infrared spectroscopy and aEEG to detect early brain hypoxicischemic injury. This management would favor performing neuroprotective measures (i.e., hypothermia) within therapeutic window. Furthermore, MRI should be considered in infants with noncardiac congenital anomalies after neonatal surgery and long-term follow-up is required to evaluate the consequences later in life.

\section{Conflict of Interest}

None.

\section{References}

1 McCann ME, Schouten AN, Dobija N, et al. Infantile postoperative encephalopathy: perioperative factors as a cause for concern. Pediatrics 2014;133(03):e751-e757

2 Stolwijk LJ, Keunen K, de Vries LS, et al. Neonatal surgery for noncardiac congenital anomalies: neonates at risk of brain injury. J Pediatr 2017;182:335-341.e1

3 de Vries LS, Groenendaal F. Patterns of neonatal hypoxic-ischaemic brain injury. Neuroradiology 2010;52(06):555-566

4 de Graaff JC, Pasma W, van Buuren S, et al. Reference values for noninvasive blood pressure in children during anesthesia: a multicentered retrospective observational cohort study. Anesthesiology 2016;125(05):904-913

5 Harteman JC, Groenendaal F, Toet MC, et al. Diffusion-weighted imaging changes in cerebral watershed distribution following neonatal encephalopathy are not invariably associated with an adverse outcome. Dev Med Child Neurol 2013;55 (07):642-653 